Diabetologe 2019 $\cdot 15: 680$

https://doi.org/10.1007/s11428-019-00525-6

Online publiziert: 19. August 2019

(c) Springer Medizin Verlag GmbH, ein Teil von Springer Nature 2019

\section{Erwiderung zum Leserbrief}

Mielck S (2019) Natriumchlorid $0,9 \%$ bei diabetischer Ketoazidose (DKA). Diabetologe. https://doi.org/10.1007/s11428-019-0524-7

\section{Originalbeitrag}

Haak T, Gölz S, Fritsche A et al (2019) Therapie des Typ-1-Diabetes. Diabetologe. https:// doi.org/10.1007/s11428-019-0458-8

Wir begrüßen den Leserbeitrag, da er doch anzeigt, dass es aktuell eine lebhafte Diskussion um die richtige Strategie der Flüssigkeitssubstitution bei diabetischer Ketoazidose (DKA) gibt.

Allerdings halten wir es für falsch, ein sog. Erratum zu publizieren, weil die in der S3-Leitlinie „Therapie des Typ-1-Diabetes" geschilderte Strategie der Gabe von 0,9\% NatriumchloridLösung $(\mathrm{NaCl})$ nach unserer ausführlichen Evidenzrecherche keinen Fehler darstellt.

Die im Jahre 2018 im New England Journal of Medicine publizierte Studie zur Flüssigkeitssubstitution bei DKA bei Kindern („PECARN DKA FLUID study group") zeigt, dass es bezüglich zerebraler Schäden keinen Unterschied macht, ob man $0,9 \%$ oder $0,45 \% \mathrm{NaCl}$ gibt [1].

Die „standards of medical care" der "American Diabetes Association" von 2018 empfehlen auch weiterhin die Gabe von isotoner Kochsalzlösung. Die Arbeit von Dhatariya et al. [2] beschreibt noch einmal länderspezifisch im Vergleich der englischen zu den amerikanischen Leitlinien die Unterschiede in der Behandlung

Thomas Haak' für Autoren der S3-Leitlinie „Therapie des Typ-1-Diabetes" der Deutschen Diabetes-Gesellschaft

' Diabetes Zentrum Mergentheim, Bad Mergentheim, Deutschland

\title{
Keine einheitlichen Behandlungsleitlinien der diabetischen Ketoazidose
}

der DKA mit Blick auf die verwendeten NaCl-Lösungen. Damit wird deutlich, dass es keine einheitlichen, international klar konsentierten Behandlungsleitlinien der DKA gibt.

Der „International Society for Pediatric and Adolescent Diabetes"(ISPAD)Konsensusbericht zur Behandlung der DKA von 2018, auf den sich auch der Leserbeitrag bezieht, bestätigt mit den dort in Abb. 2 gemachten Empfehlungen zum Behandlungsalgorithmus, dass die initiale Verwendung von $0,9 \% \mathrm{NaCl}-$ Lösung zum Start das korrekte Vorgehen darstellt und dann entsprechend der klinischen Beurteilung der Elektrolytkontrollen im weiteren Verlauf auch eine Infusion mit niedrigerer Natriumkonzentration gewählt werden kann [3].

Der Leser beschreibt korrekterweise, dass, wie im ISPAD-Konsensuspapier ausgeführt, größere Mengen an Chlorid (zugeführt über die $0,9 \% \mathrm{NaCl}$ Lösung) einen übersäuernden Effekt haben und man, sofern man nur den „base excess" überwachen würde, eine bereits vollständig rekompensierte DKA nicht erkennen und stattdessen von einer noch weiter bestehenden DKA ausgehen würde. Diese Gefahr einer metabolischen Azidose aufgrund einer Hyperchlorämie ist bekannt, tritt aber bei intakter Nierenfunktion eher selten auf und meist auch nur bei sehr hohen Infusionsmengen. Durch parallele Bedside-Plasmahydroxybutyratmessungen kann man hier zweifelsfrei erkennen, dass die DKA rekompensiert ist.

Zusammenfassend stellen wir fest, dass es bislang keine präzisen und eindeutigen, international konsentierten
Therapiealgorithmen zur Frage gibt, welche Tonizität der Flüssigkeitssubstitution zur Volumenrekonstitution verwendet soll. Wir begrüßen daher die Anmerkungen des Lesers und unterstützen die Idee, die Frage der Flüssigkeitssubstitution interdisziplinär weiter zu diskutieren.

\section{Korrespondenzadresse}

Prof. Dr. med. Thomas Haak

Diabetes Zentrum Mergentheim

Bad Mergentheim, Deutschland

haak@diabetes-zentrum.de

Interessenkonflikt. T. Haak gibt an, dass kein Interessenkonflikt besteht.

\section{Literatur}

1. Kuppermann $\mathrm{N}$ et al (2018) Clinical trial of fluid infusion rates for pediatric diabetic ketoacidosis. N Engl J Med 378:2275-2287. https://doi.org/10. 1056/NEJMoa1716816

2. Dhatariya K, Vellanki P (2017) Treatment of diabetic ketoacidosis (DKA)/hyperglycemic hyperosmolar state (HHS): novel advances in the management of hyperglycemic crises (UK versus USA). Curr Diab Rep 17:33

3. Wolfsdorf JI et al (2018) ISPAD Clinical Practice Consensus Guidelines 2018: diabetic ketoacidosis and the hyperglycemichyperosmolarstate. Pediatr Diabetes 19(Suppl. 27):155-177 